\title{
An empirical almost sure central limit theorem under the weak dependence assumptions and its application to copula processes
}

\begin{abstract}
Let: $\mathbf{Y}=\left(\mathbf{Y}_{i}\right)$, where $\mathbf{Y}_{i}=\left(Y_{i, 1}, \ldots, Y_{i, d}\right), i=1,2, \ldots$, be a $d$ dimensional, identically distributed, stationary, centered process with uniform marginals and a joint $\operatorname{cdf} F$, and $F_{n}(\mathbf{x}):=\frac{1}{n} \sum_{i=1}^{n} \mathbb{I}\left(Y_{i, 1} \leq x_{1}, \ldots, Y_{i, d} \leq x_{d}\right)$ denote the corresponding empirical cdf. In our work, we prove the almost sure central limit theorem for an empirical process $B_{n}=\sqrt{n}\left(F_{n}-F\right)$ under some weak dependence conditions due to Doukhan and Louhichi. Some application of the established result to copula processes is also presented.
\end{abstract}

1. Introduction. Since the publication of the celebrated papers by Brosamler [2], Lacey and Philipp [19] and Schatte [26]-[27], much attention has been drawn by the almost sure versions of distributional limit theorems, commonly referred to as the almost sure central limit theorems (ASCLTs). The following property is investigated in the research devoted to the ASCLTs. Namely, suppose that: $X_{1}, X_{2}, \ldots, X_{k}, \ldots$ are some r.v.'s, $f_{1}, f_{2}, \ldots, f_{k}, \ldots$ denote some real-valued measurable functions, defined on

2010 Mathematics Subject Classification. 60F15, 60F05, 60F17.

Key words and phrases. Almost sure central limit theorem, weak dependence, empirical processes, copulas. 
$\mathbb{R}, \mathbb{R}^{2}, \ldots, \mathbb{R}^{k}, \ldots$, respectively; we seek conditions under which the following almost sure (a.s.) convergence holds for some nondegenerate cdf $G$

$$
\lim _{N \rightarrow \infty} \frac{1}{W_{N}} \sum_{n=1}^{N} w_{n} \mathbb{I}\left(f_{n}\left(X_{1}, \ldots, X_{n}\right) \leq x\right)=G(x) \quad \text { a.s. },
$$

for all $x \in C_{G}$, where: $\left(w_{n}\right)$ is some sequence of weights, $W_{N}=\sum_{n=1}^{N} w_{n}$, II stands for the indicator function, and $C_{G}$ denotes the set of continuity points of $G$.

The subject matter of ASCLTs has gained immense popularity throughout the past two decades or so, and a vast number of works, where (1.1) is proved for different kinds of functions $f_{n}$ and various types of random sequences $\left(X_{n}\right)$ have been published since the early 1990's. We cite in this context the articles by: Berkes and Csáki [1], Chen and Lin [3], Cheng et al. [4], Csáki and Gonchigdanzan [5], Dudziński [9]-[10], Dudziński and Górka [11], Gonchigdanzan and Rempała [18], Matuła [20], Mielniczuk [21], Peligrad and Shao [22], Stadtmüller [28], and Zhao et al. [33], among others. The functions $f_{n}$ included, for different kinds of r.v.'s, e.g.: the partial sums (see: [1], [9], [20]-[22]), the products of partial sums (see [18]), the maxima (see: [1], [3]-[5]), the extreme order statistics (see [28]), the maxima of sums (see: [1], [11]), and - jointly - the maxima and sums as well (see: [10], [33]). It is worth mentioning that, in some cases, the indicator function $\mathbb{I}$ in (1.1) may be replaced by a larger class of functions, see, e.g., Fazekas and Rychlik [12]. We say about the functional almost sure central limit theorem then.

The principal purpose of our paper is to prove an empirical ASCLT, which comprises the case where some normalized empirical process is considered. We shall introduce the following notations. We denote by $\mathbf{Y}=\left(\mathbf{Y}_{i}\right)=$ $\left(\left(Y_{i, 1}, \ldots, Y_{i, d}\right)\right), i=1,2, \ldots$, a $d$-dimensional, identically distributed, stationary, centered process with uniform marginal cdfs and a joint cdf $F$ and by $F_{n}$, the corresponding empirical cdf, i.e., the function such that, for any $\mathbf{x}=\left(x_{1}, \ldots, x_{d}\right) \in[0,1]^{d}$,

$$
F_{n}(\mathbf{x}):=\frac{1}{n} \sum_{i=1}^{n} \mathbb{I}\left(\mathbf{Y}_{i} \leq \mathbf{x}\right)=\frac{1}{n} \sum_{i=1}^{n} \mathbb{I}\left(Y_{i, 1} \leq x_{1}, \ldots, Y_{i, d} \leq x_{d}\right)
$$

Furthermore, we define an empirical process $B_{n}$ by

$$
B_{n}(\mathbf{x}):=\sqrt{n}\left(F_{n}(\mathbf{x})-F(\mathbf{x})\right) .
$$

We also assume that a process $\left(\mathbf{Y}_{i}\right)$ satisfies the $\eta$-weak dependence assumption according to the condition stated in the work by Doukhan and Louhichi [6]. Before we cite this condition, we shall introduce some additional notations. Namely, we define the Lipschitz modulus of a real function $h$ on $\mathbb{R}^{d}$ 
as

$$
\operatorname{Lip}(h):=\sup _{\mathbf{x} \neq \mathbf{y}} \frac{|h(\mathbf{x})-h(\mathbf{y})|}{\|\mathbf{x}-\mathbf{y}\|_{1}},
$$

where $\|\mathbf{c}\|_{1}:=\left\|\left(c_{1}, \ldots, c_{d}\right)\right\|_{1}=\sum_{i=1}^{d}\left|c_{i}\right|$.

In addition, we denote by $\Lambda^{(1)}$ the set of functions that are bounded by 1 and have finite Lipschitz modulus. Finally, we shall refer to the sequences of indices $i_{1} \leq \cdots \leq i_{u}$ and $j_{1} \leq \cdots \leq j_{v}$ as $r$-distant, if $i_{u} \leq j_{1}$ and $j_{1}-i_{u}=r$.

We are now in a position to cite the weak dependence condition, originally stemming - as has been mentioned - from Doukhan and Louhichi [6].

Definition 1.1. Let $\eta=\left(\eta_{r}\right)_{r>0}$ be a real, positive sequence decreasing to zero. We say that a $d$-dimensional process $\left(\xi_{i}\right)_{i \in \mathbb{Z}}$ is $\left(\Psi, \Lambda^{(1)}, \eta\right)$-weakly dependent, if for any $r$-distant finite sequences $\mathbf{i}=\left(i_{1}, \ldots, i_{u}\right)$ and $\mathbf{j}=$ $\left(j_{1}, \ldots, j_{v}\right)$ and any functions $h_{1}, h_{2}$ in $\Lambda^{(1)}$, defined on $\mathbb{R}^{u}, \mathbb{R}^{v}$, respectively, we have

$$
\left|\operatorname{Cov}\left(h_{1}\left(\xi_{i_{1}}, \ldots, \xi_{i_{u}}\right), h_{2}\left(\xi_{j_{1}}, \ldots, \xi_{j_{v}}\right)\right)\right| \leq \Psi\left(h_{1}, h_{2}, u, v\right) \eta_{r} .
$$

For the examples of the $d$-dimensional processes satisfying the cited weak dependence condition with appropriate $\Psi, \Lambda^{(1)}$ and $\eta$, we refer the reader to Doukhan et al. [7].

Our goal is to show that, under suitable weak dependence assumptions on the process $\mathbf{Y}=\left(\mathbf{Y}_{i}\right)$, the following convergence is satisfied in $D\left([0,1]^{d}, d_{S}\right)$ - the space of cadlag functions endowed with the Skohorod metrics,

$$
\lim _{N \rightarrow \infty} \frac{1}{\log N} \sum_{n=1}^{N} \frac{1}{n} \mathbb{I}\left(B_{n}(\mathbf{x}) \leq z\right)=\mathbb{P}(\mathbb{B}(\mathbf{x}) \leq z) \quad \text { a.s. }
$$

for any $\mathbf{x} \in[0,1]^{d}$ and any $z \in \mathbb{R}$, where $B_{n}$ is an empirical process defined by (1.3) and $\mathbb{B}$ is a centered, Gaussian process, such that, for any vectors $\mathbf{v}, \mathbf{w} \in[0,1]^{d}$,

$$
\operatorname{Cov}(\mathbb{B}(\mathbf{v}), \mathbb{B}(\mathbf{w}))=\sum_{i \in \mathbb{N}} \operatorname{Cov}\left(\mathbf{Y}_{1} \leq \mathbf{v}, \mathbf{Y}_{i} \leq \mathbf{w}\right) .
$$

History of the empirical processes theory dates back to the 1930's and 1940 's, when the study of the empirical distribution functions $F_{n}(\mathbf{x})$ and the corresponding empirical processes $B_{n}(\mathbf{x})=\sqrt{n}\left(F_{n}(\mathbf{x})-F(\mathbf{x})\right)$ began. The two basic assertions concerning $F_{n}$ and $B_{n}$ are the Glivenko-Cantelli theorem and the Donsker theorem. The first of the mentioned results states that if $X_{1}, \ldots, X_{n}$ are i.i.d. real-valued r.v.'s with cdf $F$, then

$$
\left\|F_{n}-F\right\|_{\infty}=\sup _{-\infty<x<\infty}\left|F_{n}(x)-F(x)\right| \stackrel{\text { a.s. }}{\rightarrow} 0,
$$

where $F_{n}$ stands for the corresponding empirical df. 
An immediate application of this claim is Kolmogorov's goodness-of-fit test. The latter result - the Donsker theorem - states that $B_{n} \Longrightarrow \mathbb{B} \equiv$ $\mathbb{U}(F)$ in $D\left(\mathbb{R},\|\cdot\|_{\infty}\right)$, where $\mathbb{U}$ denotes a standard Brownian bridge process on $[0,1]$, i.e., it is a zero-mean Gaussian process with the covariance given by $\mathbb{E}(\mathbb{U}(s) \mathbb{U}(t))=\min (s, t)-s t$. The convergence $\Longrightarrow$ means that

$$
\mathbb{E} g\left(B_{n}\right) \rightarrow \mathbb{E} g(\mathbb{B}) \text { and } g\left(B_{n}\right) \stackrel{d}{\rightarrow} g(\mathbb{B}),
$$

for any bounded and continuous function $g: D\left(\mathbb{R},\|\cdot\|_{\infty}\right) \rightarrow \mathbb{R}$, where $\stackrel{d}{\rightarrow}$ stands for the convergence in distribution.

In the 1950's and 1960's a need for generalizations of both of the cited theorems has naturally arisen. In particular, it became clear that if a more general sample space $\Pi$ (such as, e.g., $\mathbb{R}^{d}$ or some function spaces) is considered, then the empirical distribution function is not so easy to deal with. Two basic questions have been stated in this context: (i) for what classes $C$ of subsets of the space $\Pi$ or collections $\mathcal{G}$ of real-valued functions on $\Pi$ does a natural extension of the Glivenko-Cantelli theorem hold?, (ii) for what classes $C$ of subsets of the space $\Pi$ or collections $\mathcal{G}$ of real-valued functions on $\Pi$ does a natural generalization of the Donsker theorem hold? The most relevant answers to these questions have been given during the 1970's in the papers by Vapnik and Chervonenkis [32] and Dudley [8] with significant contributions in the 1970's, 1980's and 1990's, due to the works by Pollard [23]-[25], Giné and Zinn [15]-[16], Giné [17], Talagrand [29], Gänssler and Stute [13] and Gänssler [14], among others. It is especially seen through the publications of David Pollard that the theory of empirical processes provides a set of powerful tools allowing to prove principal assertions in the field of asymptotic statistics. In view of the importance of the theory of modern empirical processes in statistics, the book of van der Vaart and Wellner [31] is worthwhile to mention as well. In its third chapter, the usefulness of this theory in statistical applications related to, i.a., the M-estimators approach, the Bootstrap methods, the Two-sample problem and Minimax theorems, has been presented in detail. For a comprehensive overview on the theory of empirical processes and their applications (e.g., in the research of asymptotic normality of M-estimators and in penalized least-squares estimation), we refer the reader to the work by van de Geer [30].

The remainder of our work is structured as follows. In Section 2, we precisely state our main result. In Section 3, we prove some lemmas, which are necessary for the proof of our claim. Section 4 contains this proof, whereas in Section 5, some application of the established proposition in terms of copula processes is depicted. Finally, in Section 6, an example of a sequence satisfying the assumptions of Theorem 2.1 is presented. This example is given in the form of Theorem 6.1. 
2. Main result. For the functions $h_{1}, h_{2}$ from $\Lambda^{(1)}$ - the class of functions defined on $\mathbb{R}^{u}, \mathbb{R}^{v}$, respectively, which are bounded by 1 and have finite Lipschitz modulus - we define the following mappings:

$$
\begin{aligned}
& \Psi_{1}\left(h_{1}, h_{2}, u, v\right):=\min (u, v)\left(\operatorname{Lip}\left(h_{1}\right)+\operatorname{Lip}\left(h_{2}\right)\right), \\
& \Psi_{2}\left(h_{1}, h_{2}, u, v\right):=\min (u, v) \operatorname{Lip}\left(h_{1}\right) \operatorname{Lip}\left(h_{2}\right), \\
& \Psi_{3}\left(h_{1}, h_{2}, u, v\right):=\left(u^{1 / 2} \operatorname{Lip}\left(h_{1}\right)+v^{1 / 2} \operatorname{Lip}\left(h_{2}\right)\right), \\
& \Psi_{4}\left(h_{1}, h_{2}, u, v\right):=(u v)^{1 / 2} \operatorname{Lip}\left(h_{1}\right) \operatorname{Lip}\left(h_{2}\right) .
\end{aligned}
$$

Our major assertion is the following ASCLT for some empirical process.

Theorem 2.1. Suppose that $\mathbf{Y}=\left(\mathbf{Y}_{i}\right)=\left(\left(Y_{i, 1}, \ldots, Y_{i, d}\right)\right), i=1,2, \ldots$, is an identically distributed, stationary, centered, $d$-dimensional process, with uniform marginal distributions and a joint $c d f F$ (thus, $Y_{i, j}^{\prime}$ s have the same uniform distribution, for any $i \in N$ and $j=1, \ldots, d)$. Let in addition: $F_{n}$ be such as in (1.2) and denote an empirical cdf of $\mathbf{Y}$, and $B_{n}$ be an empirical process defined by (1.3). Moreover, assume that there exists a constant $C>0$, such that:

(i) $\mathbf{Y}$ is $\left(\Psi_{1}, \Lambda^{(1)}, \eta_{Y, r}\right)$-weakly dependent,

or

(ii) $\mathbf{Y}$ is $\left(\Psi_{2}, \Lambda^{(1)}, \eta_{Y, r}\right)$-weakly dependent,

or

(iii) $\mathbf{Y}$ is $\left(\Psi_{3}, \Lambda^{(1)}, \eta_{Y, r}\right)$-weakly dependent, or

(iv) $\mathbf{Y}$ is $\left(\Psi_{4}, \Lambda^{(1)}, \eta_{Y, r}\right)$-weakly dependent,

with the weak dependence coefficient satisfying $\eta_{Y, r} \leq \frac{C^{2} r^{-2 \alpha}}{9 d}$ for some $\alpha>$ $d+\sqrt{1+d^{2}}$. Assume in addition that

$$
\sup _{|j-i|=r}\left|\mathbb{P}\left(\mathbf{Y}_{i} \leq \mathbf{x}, \mathbf{Y}_{j} \leq \mathbf{x}\right)-\mathbb{P}\left(\mathbf{Y}_{i} \leq \mathbf{x}\right) \mathbb{P}\left(\mathbf{Y}_{j} \leq \mathbf{x}\right)\right|=\mathcal{O}\left(r^{-1}\right)
$$

if $r \rightarrow \infty$. Then, convergence (1.5) holds true in $D\left([0,1]^{d}, d_{S}\right)$.

Observe that all of the functions $\Psi_{1}-\Psi_{4}$ may be written in the form

$$
\Psi\left(h_{1}, h_{2}, u, v\right)=c(u, v) \mu\left(\operatorname{Lip}\left(h_{1}\right), \text { Lip }\left(h_{2}\right)\right),
$$

where $c$ is some function defined on $\mathbb{N}^{2}$ and $\mu$ is a locally bounded function on $\mathbb{R}_{+}^{2}$.

Furthermore, it is easy to check that $\Psi_{1}-\Psi_{4}$ may be bounded by

$$
\Psi\left(h_{1}, h_{2}, u, v\right)=(u+v)^{s}\left(\operatorname{Lip}\left(h_{1}\right)+\operatorname{Lip}\left(h_{2}\right)\right)^{t},
$$

for some $s>0$ and some $t \in[0,2]$, since:

$$
\begin{aligned}
& \Psi_{1}\left(h_{1}, h_{2}, u, v\right) \leq(u+v)\left(\operatorname{Lip}\left(h_{1}\right)+\operatorname{Lip}\left(h_{2}\right)\right), \\
& \Psi_{2}\left(h_{1}, h_{2}, u, v\right) \leq(u+v)\left(\operatorname{Lip}\left(h_{1}\right)+\operatorname{Lip}\left(h_{2}\right)\right)^{2},
\end{aligned}
$$




$$
\begin{aligned}
& \Psi_{3}\left(h_{1}, h_{2}, u, v\right) \leq(u+v)^{1 / 2}\left(\operatorname{Lip}\left(h_{1}\right)+\operatorname{Lip}\left(h_{2}\right)\right), \\
& \Psi_{4}\left(h_{1}, h_{2}, u, v\right) \leq(u+v)\left(\operatorname{Lip}\left(h_{1}\right)+\operatorname{Lip}\left(h_{2}\right)\right)^{2} .
\end{aligned}
$$

Therefore, if $\mathbf{Y}$ is either $\left(\Psi_{1}, \Lambda^{(1)}, \eta_{Y, r}\right)$ or $\left(\Psi_{2}, \Lambda^{(1)}, \eta_{Y, r}\right)$ or $\left(\Psi_{3}, \Lambda^{(1)}, \eta_{Y, r}\right)$ or $\left(\Psi_{4}, \Lambda^{(1)}, \eta_{Y, r}\right)$-weakly dependent, then it is also $\left(\Psi_{5}, \Lambda^{(1)}, \eta_{Y, r}\right)$-weakly dependent with

$$
\Psi_{5}\left(h_{1}, h_{2}, u, v\right)=(u+v) \max \left(\operatorname{Lip}\left(h_{1}\right)+\operatorname{Lip}\left(h_{2}\right),\left(\operatorname{Lip}\left(h_{1}\right)+\operatorname{Lip}\left(h_{2}\right)\right)^{2}\right) .
$$

This fact allows, e.g., to prove the weak convergence of the corresponding sequence of $\left(\Psi_{5}, \Lambda^{(1)}, \eta_{Y, r}\right)$-weakly dependent processes (see, e.g., Proposition 1 in Doukhan and Louhichi [6]).

In addition, it is seen from Lemma 10 in [6] that some classes of Markov chains form the sequences of $\left(\Psi_{6}, \Lambda^{(1)}, \theta_{r}\right)$-weakly dependent sequences with $\Psi_{6}$ satisfying

$$
\begin{aligned}
\Psi_{6}\left(h_{1}, h_{2}, u, v\right) & \leq 2 \min \left(u \operatorname{Lip}\left(h_{1}\right), v \operatorname{Lip}\left(h_{2}\right)\right) \\
& \leq u \operatorname{Lip}\left(h_{1}\right)+v \operatorname{Lip}\left(h_{2}\right) \\
& \leq(u+v)\left(\operatorname{Lip}\left(h_{1}\right)+\operatorname{Lip}\left(h_{2}\right)\right) \\
& \leq \Psi_{5}\left(h_{1}, h_{2}, u, v\right)
\end{aligned}
$$

and hence, these Markov chains are $\left(\Psi_{5}, \Lambda^{(1)}, \theta_{r}\right)$-weakly dependent as well.

In the subsequent section, we state and prove two lemmas, which are needed for the proof of Theorem 2.1.

3. Auxiliary results. The following lemma will be used in the proof of our main result.

Lemma 3.1. Under the notations and assumptions of Theorem 2.1, we have for any $\mathbf{x} \in[0,1]^{d}$ and any Lipschitz and bounded by 1 function $g$

$$
\left|\operatorname{Cov}\left(g\left(B_{k}(\mathbf{x})\right), g\left(B_{n}(\mathbf{x})\right)\right)\right|=\mathcal{O}\left(\frac{1}{k^{3 / 2}}+\left(\frac{k}{n}\right)^{1 / 2}\right),
$$

if $k \rightarrow \infty$, provided that $k<n$.

Proof. Let $\mathbf{Y}=\left(\mathbf{Y}_{i}\right)=\left(\left(Y_{i, 1}, \ldots, Y_{i, d}\right)\right)$ be a $d$-dimensional, identically distributed, stationary, centered process with uniform marginal cdfs, a common joint $\operatorname{cdf} F$ and the corresponding empirical cdf $F_{n}$ (i.e., $F, F_{n}$ are such that $F(\mathbf{x}):=\mathbb{P}\left(Y_{i, 1} \leq x_{1}, \ldots, Y_{i, d} \leq x_{d}\right)$, for any $i \in \mathbb{N}$, where $\mathbf{x}=\left(x_{1}, \ldots, x_{d}\right) \in$ $\mathbb{R}^{d}$, and $F_{n}$ is given by (1.2)). Due to a definition of an empirical process 
$B_{n}$ in (1.3), we have, for any $\mathbf{x} \in \mathbb{R}^{d}$,

$$
\begin{aligned}
B_{n}(\mathbf{x}) & =\sqrt{n}\left(F_{n}(\mathbf{x})-F(\mathbf{x})\right)=\sqrt{n}\left(\frac{1}{n} \sum_{i=1}^{n} \mathbb{I}\left(\mathbf{Y}_{i} \leq \mathbf{x}\right)-F(\mathbf{x})\right) \\
& =\sqrt{n}\left(\frac{1}{n} \sum_{i=1}^{n}\left\{\mathbb{I}\left(\mathbf{Y}_{i} \leq \mathbf{x}\right)-\mathbb{P}\left(\mathbf{Y}_{i} \leq \mathbf{x}\right)\right\}\right) \\
& =\frac{1}{\sqrt{n}} \sum_{i=1}^{n}\left\{\mathbb{I}\left(\mathbf{Y}_{i} \leq \mathbf{x}\right)-\mathbb{P}\left(\mathbf{Y}_{i} \leq \mathbf{x}\right)\right\} \\
& =\frac{1}{\sqrt{n}} \sum_{i=1}^{n}\left\{\mathbb{I}\left(\mathbf{Y}_{i} \leq \mathbf{x}\right)-F(\mathbf{x})\right\} \\
& =\frac{S_{n}(\mathbf{x})}{\sqrt{n}}:=\frac{S_{n}}{\sqrt{n}},
\end{aligned}
$$

where

$$
S_{n}=S_{n}(\mathbf{x}):=\sum_{i=1}^{n}\left\{\mathbb{I}\left(\mathbf{Y}_{i} \leq \mathbf{x}\right)-\mathbb{P}\left(\mathbf{Y}_{i} \leq \mathbf{x}\right)\right\}=\sum_{i=1}^{n}\left\{\mathbb{I}\left(\mathbf{Y}_{i} \leq \mathbf{x}\right)-F(\mathbf{x})\right\} .
$$

For each $k \in \mathbb{N}$, we shall introduce the following notation

$$
\begin{aligned}
& Z\left(f_{k},(1, \ldots, k), \mathbf{x}\right):=\frac{S_{k}}{\sqrt{k}}=\frac{1}{\sqrt{k}} \sum_{i=1}^{k}\left\{\mathbb{I}\left(\mathbf{Y}_{i} \leq \mathbf{x}\right)-\mathbb{P}\left(\mathbf{Y}_{i} \leq \mathbf{x}\right)\right\} \\
& =\frac{1}{\sqrt{k}} \sum_{i=1}^{k}\left\{\mathbb{I}\left(\mathbf{Y}_{i} \leq \mathbf{x}\right)-F(\mathbf{x})\right\}=f_{k}\left(\mathbb{I}\left(\mathbf{Y}_{1} \leq \mathbf{x}\right), \ldots, \mathbb{I}\left(\mathbf{Y}_{k} \leq \mathbf{x}\right)\right),
\end{aligned}
$$

where

$$
f_{k}\left(z_{1}(\mathbf{x}), \ldots, z_{k}(\mathbf{x})\right):=\frac{1}{\sqrt{k}} \sum_{i=1}^{k}\left(z_{i}(\mathbf{x})-F(\mathbf{x})\right) .
$$

Furthermore, we define the term $Z\left(f_{n},(2 k+1, \ldots, 2 k+n)\right.$, x $)$ by

$$
\begin{aligned}
Z & \left(f_{n},(2 k+1, \ldots, 2 k+n), \mathbf{x}\right):=\frac{S_{2 k+n}-S_{2 k}}{\sqrt{n}} \\
& =\frac{1}{\sqrt{n}} \sum_{i=2 k+1}^{2 k+n}\left\{\mathbb{I}\left(\mathbf{Y}_{i} \leq \mathbf{x}\right)-F(\mathbf{x})\right\} \\
& =f_{n}\left(\mathbb{I}\left(\mathbf{Y}_{2 k+1} \leq \mathbf{x}\right), \ldots, \mathbb{I}\left(\mathbf{Y}_{2 k+n} \leq \mathbf{x}\right)\right),
\end{aligned}
$$

where

$$
f_{n}\left(z_{1}(\mathbf{x}), \ldots, z_{n}(\mathbf{x})\right):=\frac{1}{\sqrt{n}} \sum_{i=1}^{n}\left(z_{i}(\mathbf{x})-F(\mathbf{x})\right)
$$


Put: $s_{i}(\mathbf{x})=s_{i}, t_{i}(\mathbf{x})=t_{i}, i=1, \ldots, l, \mathbf{s}=\left(s_{1}, \ldots, s_{l}\right), \mathbf{t}=\left(t_{1}, \ldots, t_{l}\right)$. We have, for any $l \in \mathbb{N}$,

$$
\begin{aligned}
\left|f_{l}\left(s_{1}, \ldots, s_{l}\right)-f_{l}\left(t_{1}, \ldots, t_{l}\right)\right| & =\left|\frac{1}{\sqrt{l}} \sum_{i=1}^{l}\left(s_{i}-t_{i}\right)\right| \leq \frac{1}{\sqrt{l}} \sum_{i=1}^{l}\left|s_{i}-t_{i}\right| \\
& =\frac{1}{\sqrt{l}}\|\mathbf{s}-\mathbf{t}\|_{1} .
\end{aligned}
$$

This and a definition of the Lipschitz modulus in (1.4) yield

$$
\operatorname{Lip}\left(f_{l}\right) \leq \frac{1}{\sqrt{l}}
$$

Our aim now is to give a bound for the covariance $\operatorname{Cov}\left(g\left(B_{k}(\mathbf{x})\right), g\left(B_{n}(\mathbf{x})\right)\right)$, where $g$ is any Lipschitz function bounded by 1 . We assume first that $\mathbf{Y}$ is $\left(\Psi_{1}, \Lambda^{(1)}, \eta_{Y, r}\right)$-weakly dependent with $\eta_{Y, r}=O\left(r^{-\alpha}\right)$ for some $\alpha>d+$ $\sqrt{1+d^{2}}$, which corresponds to the case (i) in the statement of Theorem 2.1. As, due to the derivation at the beginning of the current proof, $B_{n}(\mathbf{x})=$ $S_{n} / \sqrt{n}$, where $S_{n}=S_{n}(\mathbf{x})=\sum_{i=1}^{n}\left\{\mathbb{I}\left(\mathbf{Y}_{i} \leq \mathbf{x}\right)-F(\mathbf{x})\right\}$, we get

$$
\left|\operatorname{Cov}\left(g\left(B_{k}(\mathbf{x})\right), g\left(B_{n}(\mathbf{x})\right)\right)\right|=\left|\operatorname{Cov}\left(g\left(\frac{S_{k}}{\sqrt{k}}\right), g\left(\frac{S_{n}}{\sqrt{n}}\right)\right)\right| .
$$

Observe that

$$
\begin{aligned}
\left|\operatorname{Cov}\left(g\left(\frac{S_{k}}{\sqrt{k}}\right), g\left(\frac{S_{n}}{\sqrt{n}}\right)\right)\right| & \leq\left|\operatorname{Cov}\left(g\left(\frac{S_{k}}{\sqrt{k}}\right), g\left(\frac{S_{2 k+n}-S_{2 k}}{\sqrt{n}}\right)\right)\right| \\
& +\left|\mathbb{E} g\left(\frac{S_{k}}{\sqrt{k}}\right)\left(g\left(\frac{S_{n}}{\sqrt{n}}\right)-g\left(\frac{S_{2 k+n}-S_{2 k}}{\sqrt{n}}\right)\right)\right| \\
& +\left|\mathbb{E} g\left(\frac{S_{k}}{\sqrt{k}}\right) \mathbb{E}\left(g\left(\frac{S_{n}}{\sqrt{n}}\right)-g\left(\frac{S_{2 k+n}-S_{2 k}}{\sqrt{n}}\right)\right)\right| \\
\text { (3.6) } & =: A_{1}+A_{2}+A_{3} .
\end{aligned}
$$

Due to the stationarity of $\mathbf{Y}$, we immediately obtain

$$
A_{3}=0 \text {. }
$$

Let us now estimate the component $A_{1}$ in (3.6). In view of (3.2)-(3.3) and the definitions of $Z$ and $f_{k}$ in (3.2), we have

$$
\begin{aligned}
& \left|\operatorname{Cov}\left(g\left(\frac{S_{k}}{\sqrt{k}}\right), g\left(\frac{S_{2 k+n}-S_{2 k}}{\sqrt{n}}\right)\right)\right| \\
& =\left|\operatorname{Cov}\left(g\left(f_{k}\left(\mathbb{I}\left(\mathbf{Y}_{1} \leq \mathbf{x}\right), \ldots, \mathbb{I}\left(\mathbf{Y}_{k} \leq \mathbf{x}\right)\right)\right), g\left(f_{n}\left(\mathbb{I}\left(\mathbf{Y}_{2 k+1} \leq \mathbf{x}\right), \ldots, \mathbb{I}\left(\mathbf{Y}_{2 k+n} \leq \mathbf{x}\right)\right)\right)\right)\right| \\
& =\left|\operatorname{Cov}\left(Z\left(g \circ f_{k},(1, \ldots, k), \mathbf{x}\right), Z\left(g \circ f_{n},(2 k+1, \ldots, 2 k+n), \mathbf{x}\right)\right)\right| .
\end{aligned}
$$

Since $\left(\mathbf{Y}_{i}\right)$ is a $d$-dimensional, $\left(\Psi_{1}, \Lambda^{(1)}, \eta_{Y, r}\right)$-weakly dependent process with a dependence coefficient $\eta_{Y, r}$ satisfying $\eta_{Y, r}=\mathcal{O}\left(r^{-\alpha}\right)$ for some $\alpha>$ 
$d+\sqrt{1+d^{2}}$, then by the proof of Lemma 2.1 in Doukhan et al. [7] and the fact that $g \circ f_{l} \in \Lambda^{(1)}$, we obtain

$$
\begin{aligned}
& \left|\operatorname{Cov}\left(g\left(\frac{S_{k}}{\sqrt{k}}\right), g\left(\frac{S_{2 k+n}-S_{2 k}}{\sqrt{n}}\right)\right)\right| \\
= & \left|\operatorname{Cov}\left(Z\left(g \circ f_{k},(1, \ldots, k), \mathbf{x}\right), Z\left(g \circ f_{n},(2 k+1, \ldots, 2 k+n), \mathbf{x}\right)\right)\right| \\
\leq & \left\{\min (k, n) \operatorname{Lip}\left(g \circ f_{k}\right)+\min (k, n) \operatorname{Lip}\left(g \circ f_{n}\right)\right\} \eta_{k},
\end{aligned}
$$

where $\eta_{k}=3\left(\eta_{Y, r} d\right)^{1 / 2}$ which - by an assumption on $\eta_{Y, r}$ in the statement of Theorem 2.1 - implies that $\eta_{k} \leq C r^{-\alpha}$ for some $\alpha>d+\sqrt{1+d^{2}}$ and some $C>0$.

Additionally, in view of (3.4) and the fact that $g$ is Lipschitz with some Lipschitz coefficient $L$, we get

$$
\begin{aligned}
\operatorname{Lip}\left(g \circ f_{l}\right) & =\sup _{\mathbf{x} \neq \mathbf{y}} \frac{\left|\left(g \circ f_{l}\right)(\mathbf{x})-\left(g \circ f_{l}\right)(\mathbf{y})\right|}{\|\mathbf{x}-\mathbf{y}\|_{1}} \\
& \leq \sup _{\mathbf{x} \neq \mathbf{y}} \frac{L\left|f_{l}(\mathbf{x})-f_{l}(\mathbf{y})\right|}{\|\mathbf{x}-\mathbf{y}\|_{1}} \leq L \sup _{\mathbf{x} \neq \mathbf{y}} \frac{\left|f_{l}(\mathbf{x})-f_{l}(\mathbf{y})\right|}{\|\mathbf{x}-\mathbf{y}\|_{1}} \\
& =L \cdot \operatorname{Lip}\left(f_{l}\right) \leq \frac{L}{\sqrt{l}} .
\end{aligned}
$$

This, the fact that $k<n$ and derivation (3.8) imply

$$
\begin{aligned}
& \left|\operatorname{Cov}\left(g\left(\frac{S_{k}}{\sqrt{k}}\right), g\left(\frac{S_{2 k+n}-S_{2 k}}{\sqrt{n}}\right)\right)\right| \\
& \quad \leq C L\left(\min (k, n) \frac{1}{\sqrt{k}}+\min (k, n) \frac{1}{\sqrt{n}}\right) \frac{1}{k^{\alpha}} \\
& \quad=\mathcal{O}\left(\frac{1}{k^{\alpha-1 / 2}}\right) \quad \text { if } k \rightarrow \infty .
\end{aligned}
$$

Thus, since $\alpha>d+\sqrt{1+d^{2}}>2$, we may write that

$$
\begin{aligned}
& \left|\operatorname{Cov}\left(g\left(\frac{S_{k}}{\sqrt{k}}\right), g\left(\frac{S_{2 k+n}-S_{2 k}}{\sqrt{n}}\right)\right)\right| \\
& =\mathcal{O}\left(\frac{1}{k^{3 / 2}}\right) \quad \text { if } k \rightarrow \infty,
\end{aligned}
$$

provided that the assumption in (i) is satisfied, i.e., $\mathbf{Y}$ is $\left(\Psi_{1}, \Lambda^{(1)}, \eta_{Y, r}\right)$ weakly dependent with $\eta_{Y, r}=\mathcal{O}\left(r^{-\alpha}\right)$ for some $\alpha>d+\sqrt{1+d^{2}}$.

By the similar reasoning as in the estimation of the expression

$$
\left|\operatorname{Cov}\left(g\left(\frac{S_{k}}{\sqrt{k}}\right), g\left(\frac{S_{2 k+n}-S_{2 k}}{\sqrt{n}}\right)\right)\right|,
$$

in the case (i), it is easy to verify that in the case (ii), i.e., when $\mathbf{Y}$ is $\left(\Psi_{2}, \Lambda^{(1)}, \eta_{Y, r}\right)$-weakly dependent with $\eta_{Y, r}=\mathcal{O}\left(r^{-\alpha}\right)$ for some $\alpha>d+$ 
$\sqrt{1+d^{2}}>2$, we have

$$
\begin{aligned}
& \left|\operatorname{Cov}\left(g\left(\frac{S_{k}}{\sqrt{k}}\right), g\left(\frac{S_{2 k+n}-S_{2 k}}{\sqrt{n}}\right)\right)\right| \\
& \leq C L \min (k, n) \frac{1}{\sqrt{k}} \frac{1}{\sqrt{n}} \frac{1}{k^{\alpha}} \\
& =\mathcal{O}\left(\frac{1}{\sqrt{n} k^{\alpha-1 / 2}}\right) \quad \text { if } k \rightarrow \infty \\
& =\mathcal{O}\left(\frac{1}{\sqrt{n} k^{3 / 2}}\right) \quad \text { if } k \rightarrow \infty \\
& =\mathcal{O}\left(\frac{1}{k^{2}}\right) \quad \text { if } k \rightarrow \infty,
\end{aligned}
$$

in the case (iii), i.e., when $\mathbf{Y}$ is $\left(\Psi_{3}, \Lambda^{(1)}, \eta_{Y, r}\right)$-weakly dependent with $\eta_{Y, r}=$ $\mathcal{O}\left(r^{-\alpha}\right)$ for some $\alpha>d+\sqrt{1+d^{2}}>2$, we obtain

$$
\begin{aligned}
& \left|\operatorname{Cov}\left(g\left(\frac{S_{k}}{\sqrt{k}}\right), g\left(\frac{S_{2 k+n}-S_{2 k}}{\sqrt{n}}\right)\right)\right| \\
& \leq C L\left(k^{1 / 2} \frac{1}{\sqrt{k}}+n^{1 / 2} \frac{1}{\sqrt{n}}\right) \frac{1}{k^{\alpha}} \\
& =\mathcal{O}\left(\frac{1}{k^{\alpha}}\right) \quad \text { if } k \rightarrow \infty \\
& =\mathcal{O}\left(\frac{1}{k^{2}}\right) \quad \text { if } k \rightarrow \infty,
\end{aligned}
$$

in the case (iv), i.e., when $\mathbf{Y}$ is $\left(\Psi_{4}, \Lambda^{(1)}, \eta_{Y, r}\right)$-weakly dependent with $\eta_{Y, r}=$ $\mathcal{O}\left(r^{-\alpha}\right)$ for some $\alpha>d+\sqrt{1+d^{2}}>2$, we get

$$
\begin{aligned}
& \left|\operatorname{Cov}\left(g\left(\frac{S_{k}}{\sqrt{k}}\right), g\left(\frac{S_{2 k+n}-S_{2 k}}{\sqrt{n}}\right)\right)\right| \\
& \leq C L(k n)^{1 / 2} \frac{1}{\sqrt{k n}} \frac{1}{k^{\alpha}} \\
& =\mathcal{O}\left(\frac{1}{k^{\alpha}}\right) \quad \text { if } k \rightarrow \infty \\
& =\mathcal{O}\left(\frac{1}{k^{2}}\right) \quad \text { if } k \rightarrow \infty .
\end{aligned}
$$

By (3.9)-(3.12), we have the following estimate for $A_{1}$ in (3.6)

$$
A_{1}=\mathcal{O}\left(\frac{1}{k^{3 / 2}}\right) \quad \text { if } k \rightarrow \infty .
$$


Thus, it remains to give the bound for the penultimate component in (3.6). Since $g$ is a bounded by 1 and Lipschitz function, we obtain

$$
\begin{aligned}
A_{2} & =\left|\mathbb{E} g\left(\frac{S_{k}}{\sqrt{k}}\right)\left(g\left(\frac{S_{n}}{\sqrt{n}}\right)-g\left(\frac{S_{2 k+n}-S_{2 k}}{\sqrt{n}}\right)\right)\right| \\
& \leq \mathbb{E}\left|g\left(\frac{S_{n}}{\sqrt{n}}\right)-g\left(\frac{S_{2 k+n}-S_{2 k}}{\sqrt{n}}\right)\right| \\
& \leq L \mathbb{E}\left|\frac{S_{n}}{\sqrt{n}}-\frac{S_{2 k+n}-S_{2 k}}{\sqrt{n}}\right|=L \mathbb{E}\left|\frac{S_{2 k+n}-S_{n}}{\sqrt{n}}-\frac{S_{2 k}}{\sqrt{n}}\right| .
\end{aligned}
$$

This and the stationarity of $\mathbf{Y}$ imply

$$
A_{2} \leq L \frac{\mathbb{E}\left|S_{2 k}\right|}{\sqrt{n}} \leq L \frac{\sqrt{\mathbb{E}\left(S_{k}\right)^{2}}}{\sqrt{n}} .
$$

Furthermore, observe that assumption (2.1) is equivalent to the following relation

$$
\begin{aligned}
C_{r, 2} & :=\sup _{|j-i|=r}\left|\operatorname{Cov}\left(\mathbb{I}\left(\mathbf{Y}_{i} \leq \mathbf{x}\right)-F(\mathbf{x}), \mathbb{I}\left(\mathbf{Y}_{j} \leq \mathbf{x}\right)-F(\mathbf{x})\right)\right| \\
& =\mathcal{O}\left(r^{-1}\right) \quad \text { if } r \rightarrow \infty,
\end{aligned}
$$

and the requirements of Theorem 1 in Doukhan and Louhichi [6] are fulfilled with $\left(X_{n}\right)=\left(\mathbb{I}\left(\mathbf{Y}_{n} \leq \mathbf{x}\right)-F(\mathbf{x})\right), q=2$ and $C_{r, q}=C_{r, 2}$. Consequently, by virtue of the mentioned theorem, we get that there exists a positive constant $M$, not depending on $k$, such that

$$
\mathbb{E}\left(S_{k}\right)^{2} \leq M k .
$$

The relations in (3.14)-(3.15) yield

$$
A_{2}=\mathcal{O}\left(\left(\frac{k}{n}\right)^{1 / 2}\right) \quad \text { if } k \rightarrow \infty .
$$

Thus, a desired relation in (3.1) follows from (3.5)-(3.7), (3.13) and (3.16).

The following claim will also be employed in the proof of Theorem 2.1.

Lemma 3.2. Under the notations (in particular for $B_{n}$ and $\mathbb{B}$ ) and assumptions of Theorem 2.1, we have

$$
B_{n} \stackrel{\mathbb{D}}{\rightarrow} \mathbb{B} \text { in } D\left([0,1]^{d}, d_{S}\right), \text { if } n \rightarrow \infty,
$$

where $\stackrel{\mathbb{D}}{\rightarrow}$ denotes the convergence in distribution.

Proof. Let: $f, g$ be the functions in $\Lambda^{(1)}$, defined on $\mathbb{R}^{u}, \mathbb{R}^{v}$, respectively, $\mathbf{i}=\left(i_{1}, \ldots, i_{u}\right), \mathbf{j}=\left(j_{1}, \ldots, j_{v}\right)$ be the sequences of natural indices, and 
$\mathbf{s}=\left(s_{1}, \ldots, s_{d}\right), \mathbf{t}=\left(t_{1}, \ldots, t_{d}\right)$ stand for the elements in $[0,1]^{d}$. With reference to a $d$-dimensional process $\mathbf{Y}$, we define:

$$
\begin{aligned}
& Z(f, \mathbf{i}, \mathbf{s}):=f\left(\mathbb{I}\left(\mathbf{Y}_{i_{1}} \leq \mathbf{s}\right), \ldots, \mathbb{I}\left(\mathbf{Y}_{i_{u}} \leq \mathbf{s}\right)\right), \\
& Z(g, \mathbf{j}, \mathbf{t}):=g\left(\mathbb{I}\left(\mathbf{Y}_{j_{1}} \leq \mathbf{t}\right), \ldots, \mathbb{I}\left(\mathbf{Y}_{j_{v}} \leq \mathbf{t}\right)\right) .
\end{aligned}
$$

By Lemma 2.1 in Doukhan et al. [7], we have that if $\mathbf{Y}$ satisfies at least one of the weak dependence conditions (i)-(iv) from Theorem 2.1, then the following property is satisfied

$$
|\operatorname{Cov}(Z(f, \mathbf{i}, \mathbf{s}), Z(g, \mathbf{j}, \mathbf{t}))| \leq(u \operatorname{Lip}(f)+v \operatorname{Lip}(g)) \eta_{r},
$$

where $\eta_{r}=3\left(\eta_{Y, r} d\right)^{1 / 2}$.

Recall that the condition $\eta_{Y, r} \leq \frac{C^{2} r^{-2 \alpha}}{9 d}$, for some $\alpha>d+\sqrt{1+d^{2}}$, implies $\eta_{r} \leq \mathrm{Cr}^{-\alpha}$ for some $\alpha>d+\sqrt{1+d^{2}}$. This and the relation in (3.18) imply that all the conditions of Theorem 1 in Doukhan et al. [7] are fulfilled and the convergence in (3.17) straightforwardly follows from this assertion.

We are now in a position to prove our main assertion. As has already been mentioned, the results stated as Lemmas 3.1-3.2 are intensively used in its proof.

4. Proof of the main result. The objective of this section is to present the proof of Theorem 2.1.

Proof. First, we will show that the following convergence holds true

$$
\lim _{N \rightarrow \infty} \frac{1}{\log N} \sum_{n=1}^{N} \frac{1}{n}\left\{\mathbb{I}\left(B_{n}(\mathbf{x}) \leq z\right)-\mathbb{E} \mathbb{I}\left(B_{n}(\mathbf{x}) \leq z\right)\right\}=0 \text { a.s. }
$$

in $D\left([0,1]^{d}, d_{S}\right)$, for any $\mathbf{x} \in[0,1]^{d}$ and any $z \in \mathbb{R}$.

By a well-known principle in the theory of the pointwise central limit theorem (see, e.g., Lacey and Philipp [19] and Berkes and Csáki [1]), in order to prove (4.1), it is enough to show that

$$
\lim _{N \rightarrow \infty} \frac{1}{\log N} \sum_{n=1}^{N} \frac{1}{n}\left\{g\left(\mathbb{I}\left(B_{n}(\mathbf{x}) \leq z\right)\right)-\mathbb{E} g\left(\mathbb{I}\left(B_{n}(\mathbf{x}) \leq z\right)\right)\right\}=0 \text { a.s. }
$$

for any Lipschitz function $g$ bounded by 1 .

By virtue of Lemma 3.1 in Csáki and Gonchigdanzan [5], in order to prove the convergence in (4.2), it is sufficient to show that

$$
\operatorname{Var}\left(\sum_{n=1}^{N} \frac{1}{n} g\left(\mathbb{I}\left(B_{n}(\mathbf{x}) \leq z\right)\right)\right)=\mathcal{O}\left(\frac{(\log N)^{2}}{(\log \log N)^{1+\varepsilon}}\right) \quad \text { if } N \rightarrow \infty,
$$

for some $\varepsilon>0$. 
Observe that

$$
\begin{aligned}
& \operatorname{Var}\left(\sum_{n=1}^{N} \frac{1}{n} g\left(\mathbb{I}\left(B_{n}(\mathbf{x}) \leq z\right)\right)\right) \leq \sum_{n=1}^{N} \frac{1}{n^{2}} \operatorname{Var}\left(g\left(\mathbb{I}\left(B_{n}(\mathbf{x}) \leq z\right)\right)\right) \\
& \quad+2 \sum_{1 \leq k<n \leq N} \frac{1}{k n}\left|\operatorname{Cov}\left(g\left(\mathbb{I}\left(B_{k}(\mathbf{x}) \leq z\right)\right), g\left(\mathbb{I}\left(B_{n}(\mathbf{x}) \leq z\right)\right)\right)\right| \\
& \quad=: \sum_{1}+\sum_{2} .
\end{aligned}
$$

Clearly,

$$
\sum_{1}<\infty
$$

Thus, it remains to estimate the component $\sum_{2}$ in (4.4). In view of Lemma 3.1, we have for $k<n$

$$
\left|\operatorname{Cov}\left(g\left(\mathbb{I}\left(B_{k}(\mathbf{x}) \leq z\right)\right), g\left(\mathbb{I}\left(B_{n}(\mathbf{x}) \leq z\right)\right)\right)\right|=\mathcal{O}\left(\frac{1}{k^{3 / 2}}+\left(\frac{k}{n}\right)^{1 / 2}\right),
$$

if $k \rightarrow \infty$. Therefore, we obtain

$$
\begin{aligned}
\sum_{2} & =\mathcal{O}\left(\sum_{1 \leq k<n \leq N} \frac{1}{k n} \frac{1}{k^{3 / 2}}+\sum_{1 \leq k<n \leq N} \frac{1}{k n}\left(\frac{k}{n}\right)^{1 / 2}\right) \\
& =\mathcal{O}\left(\sum_{k=1}^{N-1} \frac{1}{k^{5 / 2}} \sum_{n=k+1}^{N} \frac{1}{n}+\sum_{k=1}^{N-1} \frac{1}{k^{1 / 2}} \sum_{n=k+1}^{N} \frac{1}{n^{3 / 2}}\right) \\
& =\mathcal{O}\left(\sum_{k=1}^{N-1} \frac{1}{k^{5 / 2}} \sum_{n=k+1}^{N} \frac{1}{n}+\sum_{k=1}^{N-1} \frac{1}{k}\right),
\end{aligned}
$$

where the last relation follows from the fact that $\sum_{n=k+1}^{N} \frac{1}{n^{1+\gamma}} \leq \frac{1}{\gamma} \frac{1}{k^{\gamma}}$, for any $\gamma>0$.

Hence, we get

$$
\sum_{2}=\mathcal{O}(\log N) \quad \text { if } N \rightarrow \infty .
$$

In view of (4.4)-(4.6), we have

$$
\operatorname{Var}\left(\sum_{n=1}^{N} \frac{1}{n} g\left(\mathbb{I}\left(B_{n}(\mathbf{x}) \leq z\right)\right)\right)=\mathcal{O}(\log N) \quad \text { if } N \rightarrow \infty .
$$

Thus, the relations in (4.3) and (4.2) are fulfilled and consequently, (4.1) holds true.

Finally, the convergence in (4.1), Lemma 3.2 and a regularity property of logarithmic mean imply the result stated in Theorem 2.1. 
In the last part of our work, we give some applications of our main result, which refer to copula processes.

5. Application to copula processes. Let $(\mathbf{Y})=\left(\mathbf{Y}_{i}\right)$ be such that $\mathbf{Y}_{i}=\left(\tilde{F}_{1}\left(X_{i, 1}\right), \ldots, \tilde{F}_{d}\left(X_{i, d}\right)\right)$, where $\tilde{F}_{1}, \ldots, \tilde{F}_{d}$ denote the marginal cdfs of $X_{i, 1}, \ldots X_{i, d}$, respectively, $i=1,2, \ldots$ Clearly, we have

$$
\begin{aligned}
F(\mathbf{u}) & :=\mathbb{P}\left(\mathbf{Y}_{i} \leq \mathbf{u}\right) \\
& =\mathbb{P}\left(\tilde{F}_{1}\left(X_{i, 1}\right) \leq u_{1}, \ldots, \tilde{F}_{d}\left(X_{i, d}\right) \leq u_{d}\right) \\
& =\mathbb{P}\left(X_{i, 1} \leq \tilde{F}_{1}^{-1}\left(u_{1}\right), \ldots, X_{i, d} \leq \tilde{F}_{d}^{-1}\left(u_{d}\right)\right) \\
& =\tilde{F}\left(\tilde{F}_{1}^{-1}\left(u_{1}\right), \ldots, \tilde{F}_{d}^{-1}\left(u_{d}\right)\right) \\
& =C\left(u_{1}, \ldots, u_{d}\right)=C(\mathbf{u}),
\end{aligned}
$$

where $\tilde{F}$ is the joint cdf of $\left(X_{i, 1}, \ldots, X_{i, d}\right)$ and $C$ denotes the corresponding copula.

Furthermore, we obtain that

$$
\begin{aligned}
F_{n}(\mathbf{u}) & :=\frac{1}{n} \sum_{i=1}^{n} \mathbb{I}\left(\mathbf{Y}_{i} \leq \mathbf{u}\right) \\
& =\frac{1}{n} \sum_{i=1}^{n} \mathbb{I}\left(\tilde{F}_{1}\left(X_{i, 1}\right) \leq u_{1}, \ldots, \tilde{F}_{d}\left(X_{i, d}\right) \leq u_{d}\right) \\
& =\frac{1}{n} \sum_{i=1}^{n} \mathbb{I}\left(X_{i, 1} \leq \tilde{F}_{1}^{-1}\left(u_{1}\right), \ldots, X_{i, d} \leq \tilde{F}_{d}^{-1}\left(u_{d}\right)\right) \\
& =\tilde{F}_{n}\left(\tilde{F}_{1}^{-1}\left(u_{1}\right), \ldots, \tilde{F}_{d}^{-1}\left(u_{d}\right)\right),
\end{aligned}
$$

where

$$
\tilde{F}_{n}(\mathbf{x}):=\frac{1}{n} \sum_{i=1}^{n} \mathbb{I}\left(\mathbf{X}_{i} \leq \mathbf{x}\right)=\frac{1}{n} \sum_{i=1}^{n} \mathbb{I}\left(X_{i, 1} \leq x_{1}, \ldots, X_{i, d} \leq x_{d}\right) .
$$

In addition, we get

$$
C_{n}(\mathbf{u}):=C_{n}\left(u_{1}, \ldots, u_{d}\right)=\tilde{F}_{n}\left(\tilde{F}_{n, 1}^{-1}\left(u_{1}\right), \ldots, \tilde{F}_{n, d}^{-1}\left(u_{d}\right)\right),
$$

where

$$
\tilde{F}_{n, j}\left(x_{j}\right):=\frac{1}{n} \sum_{i=1}^{n} \mathbb{I}\left(X_{i, j} \leq x_{j}\right)
$$


It follows from the Glivenko-Cantelli theorem that

$$
\begin{aligned}
& \text { (5.4) } \sup _{\mathbf{u} \in[0,1]^{d}}\left|F_{n}(\mathbf{u})-C_{n}(\mathbf{u})\right| \\
& =\sup _{\mathbf{u} \in[0,1]^{d}}\left|\tilde{F}_{n}\left(\tilde{F}_{1}^{-1}\left(u_{1}\right), \ldots, \tilde{F}_{d}^{-1}\left(u_{d}\right)\right)-\tilde{F}_{n}\left(\tilde{F}_{n, 1}^{-1}\left(u_{1}\right), \ldots, \tilde{F}_{n, d}^{-1}\left(u_{d}\right)\right)\right| \stackrel{\text { a.s. }}{\rightarrow} 0,
\end{aligned}
$$

provided that $n \rightarrow \infty$.

Let: $F, C, F_{n}, C_{n}$ be such as in (5.1)-(5.3), respectively. Put

$$
B_{n}(\mathbf{u}):=\sqrt{n}\left(F_{n}(\mathbf{u})-F(\mathbf{u})\right) .
$$

By (5.1)-(5.3), we may rewrite $B_{n}(\mathbf{u})$ as follows

$$
\begin{aligned}
B_{n}(\mathbf{u}) & =\sqrt{n}\left(F_{n}(\mathbf{u})-F(\mathbf{u})\right)=\sqrt{n}\left(F_{n}(\mathbf{u})-C(\mathbf{u})\right) \\
& =\sqrt{n}\left(F_{n}(\mathbf{u})-C_{n}(\mathbf{u})\right)+\sqrt{n}\left(C_{n}(\mathbf{u})-C(\mathbf{u})\right) .
\end{aligned}
$$

By Theorem 2 in Doukhan et al. [7], we have that if a copula $C$ has continuous first partial derivatives, then

$$
\sqrt{n}\left(C_{n}-C\right) \stackrel{\mathbb{D}}{\rightarrow} \mathbb{G} \quad \text { in } D\left([0,1]^{d}, d_{S}\right), \text { if } n \rightarrow \infty,
$$

where the Gaussian limit $\mathbb{G}$ has continuous sample paths of the form

$$
\mathbb{G}(\mathbf{u})=\mathbb{B}(\mathbf{u})-\sum_{j=1}^{d} \frac{\partial C}{\partial u_{j}}(\mathbf{u}) \mathbb{B}\left(\mathbf{v}_{j}\right),
$$

with $\mathbf{v}_{j} \in[0,1]^{d}$ standing for the vector having - except for the $j$ th coordinate - all the coordinates equal to $u_{j}$.

Combining (5.4)-(5.6), we obtain

$$
B_{n}(\mathbf{u}) \stackrel{\mathbb{D}}{\rightarrow} \mathbb{G} \quad \text { in } D\left([0,1]^{d}, d_{S}\right), \text { if } n \rightarrow \infty,
$$

and hence, the conclusion identical with that of Lemma 3.2 holds true.

Therefore, if the process $\mathbf{Y}=\left(\mathbf{Y}_{i}\right)=\left(\left(Y_{i, 1}, \ldots, Y_{i, d}\right)\right), i=1,2, \ldots$, satisfies one of the weak dependence assumptions from Theorem 2.1, it follows from Lemmas 3.1-3.2 and the proof of Theorem 2.1 that

$$
\lim _{N \rightarrow \infty} \frac{1}{\log N} \sum_{n=1}^{N} \frac{1}{n} \mathbb{I}\left(B_{n}(\mathbf{x}) \leq z\right)=\mathbb{P}(\mathbb{B}(\mathbf{x}) \leq z) \quad \text { a.s. }
$$

where $B_{n}$ is defined such as in (5.5).

6. An example of a sequence satisfying the assumptions of Theorem 2.1. Below, we provide an example of a sequence, which satisfies the assumptions of our main result. Let us formulate the following assertion. 
Theorem 6.1. Suppose that $\mathbf{Y}=\left(\mathbf{Y}_{i}\right)=\left(\left(Y_{i, 1}, \ldots, Y_{i, d}\right)\right), i=1,2, \ldots$ is an identically distributed, stationary, centered, d-dimensional process, with uniform marginal distributions and a joint cdf $F$ (thus, $Y_{i, j}^{\prime}$ s have the same uniform distribution, for any $i \in N$ and $j=1, \ldots, d)$. Let in addition $F_{n}$ be such as in (1.2) and denote an empirical cdf of $\mathbf{Y}$, and $B_{n}$ be an empirical process defined by (1.3). Furthermore, assume that:

$$
\begin{gathered}
\lim _{n \rightarrow \infty} \frac{\sigma_{n, j}}{\sqrt{n}}=\sigma \quad \text { for any } j=1, \ldots, d, \\
\max _{1 \leq j \leq d} \mathbb{E}\left(S_{n, j}-\mathbb{E} S_{n, j}\right)^{2+\delta}=\mathcal{O}\left(n^{1+\delta / 2}\right),
\end{gathered}
$$

as $n \rightarrow \infty$, for some $\delta>0$, where: $S_{n, j}:=Y_{1, j}+Y_{2, j}+\cdots+Y_{n, j}, \sigma_{n, j}:=$ $\sqrt{\mathbb{D}^{2}\left(S_{n, j}\right)^{2}}$, and that $\left(\zeta_{n}\right)$ forms a sequence of i.i.d. r.v.'s, such that for any $j=1, \ldots, d$, the distribution of $Y_{1, j}$ is independent of $\left(\zeta_{n}\right)$ and that, there exists a measurable function $G$ satisfying the following conditions:

$$
Y_{n+1, j}=G\left(Y_{n, j}, \ldots, Y_{n-d+1, j}, \zeta_{n}\right) \quad \text { for any } j=1, \ldots, d,
$$

and

$$
\begin{aligned}
\|G(0, \zeta)\|_{m}:= & \left(\mathbb{E}|G(0, \zeta)|^{m}\right)^{1 / m}<\infty, \\
\|G(x, \zeta)-G(y, \zeta)\|_{m} & :=\left(\mathbb{E}|G(x, \zeta)-G(y, \zeta)|^{m}\right)^{1 / m} \\
& \leq \beta^{m}|x-y|^{m},
\end{aligned}
$$

for some $0 \leq \beta<1$ and $m \geq 1$.

Finally, let $\mathbb{E}\left|Y_{1}\right|:=\mathbb{E}\left|Y_{1, j}\right|$, for any $j=1, \ldots, d$, satisfy, for some positive constant $C$,

(6.6) $\beta^{r} \mathbb{E}\left|Y_{1}\right| \leq \frac{C^{2} r^{-2 \alpha}}{18 d} \quad$ for some $\alpha>d+\sqrt{1+d^{2}}$ and $\beta$ as in (6.5).

Then, (1.5) holds true for $\mathbf{Y}$ in $D\left([0,1]^{d}, d_{S}\right)$.

Proof. It is easily seen from Lemma 10 in Doukhan and Louhichi [6] and Subsection 2.2.1 in Doukhan et al. [7] that if the conditions in (6.3)-(6.5) are fulfilled, then a $d$-dimensional process $\mathbf{Y}$ is $\left(\Psi_{5}, \Lambda, \tilde{\eta}_{Y, r}\right)$-weakly dependent, where:

$$
\begin{gathered}
\Psi_{5}\left(h_{1}, h_{2}, u, v\right):=2 \min \left(u\left\|h_{1}\right\|_{\infty} \operatorname{Lip}\left(h_{1}\right), v\left\|h_{2}\right\|_{\infty} \operatorname{Lip}\left(h_{2}\right)\right), \\
\Lambda:=\bigcup_{n=1}^{\infty} \Lambda_{n}, \text { where } \Lambda_{n}:=\left\{h: \mathbb{R}^{n} \rightarrow \mathbb{R}: \operatorname{Lip}(h)<\infty\right\},
\end{gathered}
$$

$\tilde{\eta}_{Y, r}=\beta^{r} \mathbb{E}\left|Y_{1}\right|$, where $\beta, \mathbb{E}\left|Y_{1}\right|$ satisfy (6.5)-(6.6), respectively.

Since $\Lambda^{(1)}$ - the class of functions that are bounded by 1 and have finite Lipschitz modulus - is a subclass of $\Lambda$, we immediately get that $\mathbf{Y}$ is also 
$\left(\Psi_{5}, \Lambda^{(1)}, \eta_{Y, r}\right)$-weakly dependent. On the other hand, let us notice that if $h_{1}, h_{2} \in \Lambda^{(1)}$, then: $\left\|h_{1}\right\|_{\infty}<\infty,\left\|h_{2}\right\|_{\infty}<\infty$, and

$$
\Psi_{5}\left(h_{1}, h_{2}, u, v\right) \leq 2 \min (u, v)\left(\operatorname{Lip}\left(h_{1}\right)+\operatorname{Lip}\left(h_{2}\right)\right)=: \tilde{\Psi}\left(h_{1}, h_{2}, u, v\right),
$$

which means that $\mathbf{Y}$ is $\left(\tilde{\Psi}, \Lambda^{(1)}, \tilde{\eta}_{Y, r}\right)$-weakly dependent as well. Consequently, it follows from the definition of the $\left(\tilde{\Psi}, \Lambda^{(1)}, \tilde{\eta}_{Y, r}\right)$-weak dependence that if $\mathbf{Y}$ is $\left(\tilde{\Psi}, \Lambda^{(1)}, \tilde{\eta}_{Y, r}\right)$-weakly dependent with

$$
\tilde{\Psi}\left(h_{1}, h_{2}, u, v\right)=2 \min (u, v)\left(\operatorname{Lip}\left(h_{1}\right)+\operatorname{Lip}\left(h_{2}\right)\right),
$$

then it is also $\left(\Psi, \Lambda^{(1)}, \eta_{Y, r}\right)$-weakly dependent with:

$$
\begin{gathered}
\Psi\left(h_{1}, h_{2}, u, v\right):=\frac{\tilde{\Psi}\left(h_{1}, h_{2}, u, v\right)}{2}=\min (u, v)\left(\operatorname{Lip}\left(h_{1}\right)+\operatorname{Lip}\left(h_{2}\right)\right), \\
\eta_{Y, r}:=2 \tilde{\eta}_{Y, r}=2 \beta^{r} \mathbb{E}\left|Y_{1}\right| .
\end{gathered}
$$

As $\mathbf{Y}$ is $\left(\Psi, \Lambda^{(1)}, \eta_{Y, r}\right)$-weakly dependent with $\Psi, \eta_{Y, r}$ such as in (6.7)-(6.8), respectively, and (6.6) holds, we obtain that all the necessary assumptions of Theorem 2.1, in particular the constraint that $\eta_{Y, r} \leq \frac{C^{2} r^{-2 \alpha}}{9 d}$, for some constant $C>0$ and some $\alpha>d+\sqrt{1+d^{2}}$, are fulfilled. Thus, in view of the mentioned Theorem 2.1, a $d$-dimensional process in the statement of Theorem 6.1 satisfies the relation in (1.5).

Acknowledgement. We wish to thank the anonymous Referee for the valuable remarks and suggestions.

\section{REFERENCES}

[1] Berkes, I., Csáki, E., A universal result in almost sure central limit theory, Stoch. Proc. Appl. 94 (2001), 105-134.

[2] Brosamler, G., An almost everywhere central limit theorem, Math. Proc. Cambridge Philos. Soc. 104 (1988), 561-574.

[3] Chen, S., Lin, Z., Almost sure max-limits for nonstationary Gaussian sequence, Statist. Probab. Lett. 76 (2006), 1175-1184.

[4] Cheng, S., Peng, L., Qi, Y., Almost sure convergence in extreme value theory, Math. Nachr. 190 (1998), 43-50.

[5] Csáki, E., Gonchigdanzan, K., Almost sure limit theorems for the maximum of stationary Gaussian sequences, Statist. Probab. Lett. 58 (2002), 195-203.

[6] Doukhan, P., Louhichi, S., A new weak dependence condition and applications to moment inequalities, Stochastic Process. Appl. 84 (1999), 314-342.

[7] Doukhan, P., Fermanian, J. D., Lang, G., An empirical central limit theorem with applications to copulas under weak dependence, Stat. Infer. Stoch. Process. 12 (2009), 65-87.

[8] Dudley, R. M., Central limit theorems for empirical measures, Ann. Probability 6 (1978), 899-929 (Correction, ibid. 7 (1979), 909-911).

[9] Dudziński, M., A note on the almost sure central limit theorem for some dependent random variables, Statist. Probab. Lett. 61 (2003), 31-40. 
[10] Dudziński, M., The almost sure central limit theorems in the joint version for the maxima and sums of certain stationary Gaussian sequences, Statist. Probab. Lett. 78 (2008), 347-357.

[11] Dudziński, M., Górka , P., The almost sure central limit theorems for the maxima of sums under some new weak dependence assumptions, Acta Math. Sin., English Series 29, (2013), 429-448.

[12] Fazekas, I., Rychlik, Z., Almost sure functional limit theorems, Ann. Univ. Mariae Curie-Skłodowska Sect. A 56 (2002), 1-18.

[13] Gänssler, P., Stute, W., Empirical Processes: A survey of results for independent and identically distributed random variables, Ann. Probab. 7 (1979), 193-243.

[14] Gänssler, P., Empirical Processes, IMS Lecture Notes - Monograph Series, vol. 3, Hayward, 1983.

[15] Giné, E., Zinn, J., Some limit theorems for empirical processes, Ann. Probab. 12 (1984), 929-989

[16] Giné, E., Zinn, J., Lectures on the central limit theorem for empirical processes, in: Probability and Banach spaces (Zaragoza, 1985), vol. 1221 of Lecture Notes in Math., 50-113, Springer, Berlin, 1986.

[17] Giné, E., Empirical processes and applications: An overview, Bernoulli 2 (1996), $1-28$.

[18] Gonchigdanzan, K., Rempała, G., A note on the almost sure limit theorem for the product of partial sums, Appl. Math. Lett. 19 (2006), 191-196.

[19] Lacey, M., Philipp, W., A note on the almost sure central limit theorem, Statist. Probab. Lett. 9 (1990), 201-205.

[20] Matuła, P., Convergence of weighted averages of associated random variables, Probab. Math. Statist. 16 (1996), 337-343.

[21] Mielniczuk, J., Some remarks on the almost sure central limit theorem for dependent sequences. In: Limit theorems in Probability and Statistics II (I. Berkes, E. Csáki, M. Csörgö, eds.), Bolyai Institute Publications, Budapest, 2002, 391-403.

[22] Peligrad, M., Shao, Q., A note on the almost sure central limit theorem for weakly dependent random variables, Statist. Probab. Lett. 22 (1995), 131-136.

[23] Pollard, D., Limit theorems for empirical processes, Zeitschrift für Wahrscheinlichkeitstheorie und verwandte Gebiete 57 (1981), 181-195.

[24] Pollard, D., A central limit theorem for empirical processes, J. Austral. Math. Soc. (Series A) 33 (1982), 235-248.

[25] Pollard, D., Empirical Processes: Theory and Applications, vol. 2 of NSF-CBMS Regional Conference Series in Probability and Statistics, IMS, Hayward, 1990.

[26] Schatte, P., On strong versions of the central limit theorem, Math. Nachr. 137 (1988), 249-256.

[27] Schatte, P., On the central limit theorem with almost sure convergence, Probab. Math. Statist. 11 (1991), 237-246.

[28] Stadtmüller, U., Almost sure versions of distributional limit theorems for certain order statistics, Statist. Probab. Lett. 58 (2002), 413-426.

[29] Talagrand, M., The Glivenko-Cantelli problem. Ten years later, J. of Theoret. Probab. 9 (1996), 371-384.

[30] van de Geer, S., Empirical Process Theory and Applications, ETH, Zürich, 2006.

[31] van der Vaart, A. W., Wellner, J. A., Weak Convergence and Empirical Processes (With Applications to Statistics), Springer, New York, 1996.

[32] Vapnik, V. N., Chervonenkis, A. Y., On the uniform convergence of relative frequencies of events to their probabilities, Theory Probab. Appl. 16 (1971), 264-280. 
[33] Zhao, S., Peng, Z., Wu, S., Almost sure convergence for the maximum and the sum of nonstationary Gaussian sequences, J. Inequal. Appl. 2010 (2010), Art. ID 856495, $14 \mathrm{pp}$.

Marcin Dudziński

Faculty of Applied Informatics and Mathematics

Department of Applied Mathematics

Warsaw University of Life Sciences

ul. Nowoursynowska 159

02-776 Warszawa

Poland

e-mail: marcin_dudzinski@sggw.pl

Received September 1, 2016 